\section{Aposição não restritiva em língua portuguesa: análise e formalização segundo a Gramática Discursivo-Funcional}

Non-restrictive apposition in Portuguese: analysis and formalization according to Functional Discourse Grammar

Márcia Teixeira NOGUEIRA (UFC) marciatn@gmail.com

Recebido em: 30 de maio de 2018. Aceito em: 10 de jul. de 2018.
NOGUEIRA, Márcia Teixeira. Aposição não restritiva em língua portuguesa: análise e formalização segundo a Gramática Discursivo-Funcional. Entrepalavras, Fortaleza, v. 8, n. esp. p. 130-152, set. 2018 .

Resumo: Este artigo trata das construções apositivas não restritivas e sua formalização segundo o modelo da Gramática Discursivo-Funcional (GDF). Tem o objetivo de interpretar, com base nesse suporte teórico, a formulação relativa aos níveis Interpessoal e Representacional no uso dessas construções. Em Nogueira (1999, 2012), argumentei que construções apositivas não restritivas assemelham-se a parênteses por não estarem diretamente relacionadas às condições de verdade das orações queas contêm, embora contribuam para o sentido global delas. Assumi que essas expressões têm seu próprio valor ilocucional, que pode ser distinto daquele da frase em que se encontram. Em Hannay e Keizer (2005), as aposições não restritivas também são analisadas como atos discursivos separados, que tanto podem ser de referência como de atribuição. Neste estudo, com base na GDF (HANNAY; KEIZER, 2005; KEIZER, 2008; HENGEVELD; MACKENZIE, 2008; HENGEVELD, 2008), apresento uma proposta classificatória para as construções apositivas não restritivas, a partir de relações textual-semânticas e funções textual-discursivas.

Palavras-chave: Aposição. Aposição não restritiva. Gramática DiscursivoFuncional. 
Abstract: This paper deals with non-restrictive apposition and their formalization according to Functional Discourse Grammar (FDG). It aims to analyze, based on this theoretical support, the formulation related to the Interpersonal and Representational levels in the use of these constructions. I argued, in Nogueira (1999, 2012), that non-restrictive appositions resemble parentheses because they are not directly related to the truth conditions of the sentences that contain them, although they contribute to their overall meaning. I assumed that these expressions have their own illocutionary value, which may be distinct from that of the sentence in which they are found. Hannay and Keizer (2008) also identify, in non-restrictive apposition, a separate discourse act that can be both reference and attribution. This paper, based on FDG (HANNAY; KEIZER, 2005; KEIZER, 2008; HENGEVELD; MACKENZIE, 2008; HENGEVELD, 2008), presents a classificatory proposal for non-restrictive apposition, based on textual-semantic relations and textual-discursive functions.

Keywords: Apposition. Non-restrictive apposition. Functional Discourse Grammar.

\section{Introdução}

O tratamento do fenômeno da aposição tem sido marcado por muitas divergências de análise que se iniciam nos diferentes critérios de identificação de uma construção apositiva. Assumo, com Matthews (1981), Quirk et al. (1985) e Meyer (1992), a existência de uma gradiência no fenômeno da aposição e abrigo nesse rótulo diferentes tipos de construções apositivas na perspectiva de que algumas são semântica e sintaticamente mais típicas do que outras. Em Nogueira (1999), afirmei que o traço comum entre as diferentes construções ditas apositivas, mesmo as mais marginais, é sua natureza centrípeta: as unidades em aposição giram em torno de um único centro, segundo define Camara Jr. (1986, p. 47).

Nessa ampla categoria, a construção dita não restritiva ocorre quando os elementos em aposição estão em unidades de informação separadas, o que é indicado, no discurso oral, por sua inclusão em unidades tonais diferentes, e, na escrita, por meio do uso da vírgula ou outra pontuação. Expressões apositivas não restritivas não estão diretamente relacionadas às condições de verdade das orações que as contêm, embora contribuam para o sentido global delas. Essa exterioridade não significa que o conteúdo de uma aposição deva ser desprezado, mas que ele contribui com seu próprio valor de verdade, para o sentido da oração em que se encontra. Com base em Blakemore (1996), assumi, também, que uma expressão apositiva não restritiva se comporta como um ato de fala distinto daquele em que se insere, tendo, portanto, seu próprio valor ilocucional. 
v. 8 (esp.)

130-152 set. 2018

Com o modelo da GDF, que toma o ato discursivo, e não a oração, como unidade básica de análise linguística, Hannay e Keizer (2005, p. 165) tratam as aposições não restritivas como um tipo particular de holofrase ${ }^{1}$. Com ela, o falante pode monitorar o conhecimento por ele pressuposto do ouvinte, ajudando-o a captar, de modo bem sucedido, os referentes discursivos, seja após a introdução desses referentes, para identificá-los mais claramente ou para elaborá-los; seja para apresentálos de uma forma alternativa, que será útil a futuras referências discursivas, seja para criar vários efeitos retóricos.

Neste artigo, com base em autores da GDF (HANNAY; KEIZER, 2005; KEIZER, 2008; HENGEVELD; MACKENZIE, 2008; HENGEVELD, 2008), apresento uma proposta classificatória para as construções apositivas não restritivas do português, retomando suas relações textual-semânticas básicas de equivalência e atribuição (QUIRK et al., 1985) e indicando-lhes funções textual-discursivas correspondentes, tais como reformulação, avaliação, exemplificação, particularização, generalização. Ilustro essa classificação com a formalização, segundo a GDF, de aposições não restritivas identificadas em textos técnicodidáticos, oratórios e dramáticos obtidos do Banco de Dados de Língua Escrita Contemporânea no Brasil, da Faculdade de Ciências e Letras, UNESP de Araraquara-SP.

O artigo se organiza em quatro seções. Na primeira, descrevo, em linhas gerais, o suporte teórico da Gramática Discursivo-Funcional; na segunda seção, discorro sobre características gerais das aposições não restritivas segundo alguns pesquisadores da GDF (HANNAY; KEIZER, 2005; KEIZER, 2008) e proponho uma tipologia com base nas classes de relações textual-semânticas e funções textual-discursivas desse tipo de aposição; na terceira seção, apresento a metodologia e os resultados desse estudo, com a interpretação e formalização de algumas ocorrências de aposições não restritivas relativas às classes propostas; por último, faço minhas considerações finais.

${ }^{1}$ Holofrases são elocuções que, na perspectiva de uma gramática voltada para a sentença, parecem incompletas, mas, numa perspectiva de uma gramática orientada para o discurso, são vistas como unidades completas, pois, no discurso informal, os falantes frequentemente se restringem a porções de informação nova e focal (HENGEVELD; MACKENZIE, 2008, p. 4). 


\section{A Gramática Discursivo-Funcional (GDF)}

Partindo de propostas de reformulação de alguns pontos da Gramática Funcional (GF) de Dik (1997), Hengeveld e Mackenzie (2008) formularam um novo modelo de gramática funcional como uma expansão de uma gramática da frase para uma gramática funcional orientada para o discurso, a Gramática Discursivo-Funcional (GDF). Os autores justificam a elaboração da nova proposta com dois argumentos centrais: a existência de muitos fenômenos linguísticos que só podem ser explicados em termos de unidades maiores que a frase em si mesma, tais como formas verbais narrativas, partículas discursivas, cadeias anafóricas; e o uso corrente de expressões linguísticas que são menores do que a frase e, no entanto, funcionam como enunciados completos e independentes no discurso.

Hengeveld e Mackenzie (2008, p. 2) apontam uma diferença essencial da GDF em relação à GF: ao contrário do modelo da GF, que é ascendente (bottom up), um modelo com a perspectiva da produção do discurso é descendente (top-down), ou seja, explicita que a geração de estruturas subjacentes e, em particular, as interfaces entre os vários níveis podem ser descritas em termos das decisões comunicativas que um falante toma quando constrói um enunciado. A construção de expressões linguísticas pode, portanto, ser interpretada como um processo de tomada de decisões por parte do falante.

Para prover adequação tipológica, psicológica e pragmática à nova teoria, Hengeveld e Mackenzie (2008) distinguem quatro componentes: Conceitual, Contextual, Gramatical e Componente de Saída. No Componente Conceitual, estão as intenções comunicativas e as conceitualizações que demandarão formulação no Componente Gramatical. No Componente Contextual, encontram-se as informações do contexto comunicativo que podem influenciar, de alguma forma, a formulação dos atos discursivos. No Componente de Saída (output), ocorre a conversão da informação fonológica em sinais acústicos, gráficos ou visuais.

É no componente Gramatical que se dão as operações gramaticais de formulação e codificação.

A operação de formulação ocorre nos níveis Interpessoal e Representacional. No nível Interpessoal, encontra-se "a maior unidade da interação relevante para a análise gramatical", o Move (M), definida como uma contribuição autônoma que "ou é ou abre a possibilidade de uma 
v. 8 (esp.)

$130-152$ set. 2018

reação". Um Move pode estar composto por um ou mais Atos Discursivos (A), que constituem "a menor unidade identificável de uma conduta comunicativa". Por sua vez, o Ato Discursivo pode ser constituído por quatro tipos diferentes de unidades: a Ilocução (ILL), os Participantes $\left(\mathrm{P}_{1} \mathrm{e}\right.$ $\mathrm{P}_{2}$ ) e o Conteúdo Comunicado (C). A Ilocução diz respeito às propriedades lexicais e formais do Ato Discursivo, que pode ser atribuído ao uso interpessoal convencionalizado na realização da intenção comunicativa. Já os participantes na interação ganham formalização na alternância dos papéis de Falante $\left(\mathrm{P}_{1}\right)$ e Ouvinte $\left(\mathrm{P}_{2}\right)$. O Conteúdo Comunicado, por sua vez, contém a totalidade do que o Falante deseja evocar na sua comunicação com o Ouvinte. Cada Conteúdo Comunicado contém um ou mais Subatos, aos quais são atribuídas funções pragmáticas. Esses subatos podem ser de dois tipos: Atributivo (T), para evocar uma propriedade; ou Referencial (R), para evocar um referente. A GDF distingue referência de designação. A designação é de natureza categorial, pois diz respeito ao conteúdo semântico de uma unidade lexical, ou seja, à propriedade de um item lexical de designar entidades ontológicas, tendo, portanto, seu domínio no nível Representacional; a referência não é uma propriedade de um item lexical; ela concerne a uma decisão do falante de fazer referência a objetos do discurso. Desse modo, a unidade um leão constitui uma representação semântica (relativa, portanto, ao nível Representacional) de uma entidade ontológica que pode ser evocada referencial (Eu vi um leão) ou atributivamente (Este animal é um leão). No primeiro caso, há instauração de um referente discursivo; já no segundo, a atribuição de uma propriedade a um animal (HENGEVELD; MACKENZIE, 2008, p. 129).

Conforme Hengeveld eMackenzie (2008), o nível Representacional está relacionado aos aspectos semânticos de uma expressão linguística. É nesse nível que se encontram as seguintes categorias semânticas essenciais à função representacional da língua: o Indivíduo (x), entidade de primeira ordem, que pode ser localizada no espaço e pode ser avaliada em termos de sua existência; o Estado-de-coisas (e), entidade de segunda ordem, que pode ser localizada no espaço e no tempo e pode ser avaliada em termo de sua realidade; o Conteúdo Proposicional (p), entidade de terceira ordem, que constitui um constructo mental, não podendo assim ser localizado nem no espaço nem no tempo, mas avaliado em termos de verdade; e a Propriedade (f), categoria que não tem existência independente e pode ser caracterizada em termos de sua aplicabilidade. Os Estados-de-coisas podem formar unidades tematicamente coerentes, os Episódios (ep). 
O nível Morfossintático inicia a consideração do processo de codificação linguística. É ele o responsável por receber o input dos outros dois níveis (Interpessoal e Representacional), fazendo-os emergir numa representação estrutural. Essa representação será codificada como um constructo fonológico no nível seguinte (nível Fonológico), que será input para o componente de articulação, o Output do modelo.

Neste artigo, a análise de ocorrências de aposições não restritivas considera suas propriedades pragmáticas e semânticas, portanto atenção especial será dada às opções relativas à formulação, isto é, aos dois primeiros níveis da GDF - Interpessoal e Representacional.

\section{Aposições não restritivas sob o olhar da GDF}

Em Hannay e Keizer (2005, p. 160), as aposições não restritivas são analisadas como unidades comunicativamente autônomas, cuja independência encontra-se subjacente à autonomia fonética, à separação pela pontuação e à independência estrutural. Rediscutindo os traços definidores da aposição não restritiva apontados por alguns autores, tais como Quirk et al. (1985), Hannay e Keizer (2005, p. 165) assumem as seguintes propriedades gerais desse tipo de aposição: a) Elementos apositivos podem ser referenciais ou não referenciais (atributivos); quando o elemento apositivo é referencial, seu referente será idêntico ao referente do núcleo (host); b) A omissão do núcleo ou da aposição terá sempre um efeito resultante. Em alguns casos, a expressão resultante será sintática ou semanticamente inaceitável; a omissão de qualquer elemento terá sempre, todavia, um efeito na interpretação e/ ou coerência da construção resultante dentro de um discurso particular; c) As aposições serão analisadas como unidades independentes do discurso; isto é, não como parte da oração matriz, embora, obviamente, se liguem a algum elemento dessa oração. Os elementos apositivos serão vistos como holofrases, sendo não sentenciais na forma, mas promovendo uma contribuição plena e completa para o discurso.

Hannay e Keizer (2005, p. 168) retomam a ideia corrente de que as construções apositivas não restritivas podem ser parafraseadas como construções copulativas, e sugerem que seria interessante poder classificálas de modo semelhante. Propõem, a partir dessa semelhança, uma classificação das aposições não restritivas de acordo com as funções que elas exercem no discurso, sugerindo três grandes classes: a identificação (identification), a justificação (justification) e a rotulação (labelling), cada 
v. 8 (esp.)

130-152 set. 2018

uma delas especificada em subtipos conforme diferenças que podem ser apreendidas nos níveis Interpessoal e Representacional. Para os autores, a representação subjacente tem correspondência com a função discursiva de uma aposição, e essa função pode determinar os vários traços formais das construções apositivas. Hannay e Keizer (2005, p. 182) sugerem os seguintes esquemas gerais para representação das aposições não restritivas nos níveis Interpessoal e Representacional da GDF: ${ }^{2}$

Quando as duas unidades (host e apposition) $\left(\mathrm{R}_{1}\right.$ e $\left.\mathrm{R}_{2}\right)$ são referenciais: ${ }^{3}$

$\left(M_{1}:\left[\left(A_{1}:\left[\operatorname{ILL}\left(\mathrm{P}_{1}\right)_{\mathrm{S}}\left(\mathrm{P}_{2}\right)_{\mathrm{A}}\left(\mathrm{C}_{1}:\left[\ldots\left(\mathrm{T}_{1}\right)\left(\mathrm{R}_{1}\right) \ldots\right]\left(\mathrm{C}_{1}\right): \Sigma^{\mathrm{C}}\right): \Sigma^{\mathrm{F}}\right]\left(\mathrm{A}_{1}\right): \Sigma^{\mathrm{A}}\right)_{\Phi}\right.\right.$ $\left.\left.\left(\mathrm{A}_{2}:\left[\operatorname{ILL}\left(\mathrm{P}_{1}\right)_{\mathrm{S}}\left(\mathrm{P}_{2}\right)_{\mathrm{A}}\left(\mathrm{C}_{2}:\left[\ldots\left(\mathrm{R}_{2}\right) \ldots\right]\left(\mathrm{C}_{2}\right): \Sigma^{\mathrm{C}}\right): \Sigma^{\mathrm{P}}\right]\left(\mathrm{A}_{2}\right): \Sigma^{\mathrm{A}}\right)_{\Phi}\right]\left(\mathrm{M}_{1}\right)\right)$

Quando a aposição $\left(\mathrm{T}_{2}\right)$ é não referencial (atributiva): $\left(\mathrm{M}_{1}:\left[\left(\mathrm{A}_{1}:\left[\operatorname{ILL}\left(\mathrm{P}_{1}\right)_{\mathrm{S}}\left(\mathrm{P}_{2}\right)_{\mathrm{A}}\left(\mathrm{C}_{1}:\left[\ldots\left(\mathrm{T}_{1}\right)\left(\mathrm{R}_{1}\right) \ldots\right]\left(\mathrm{C}_{1}\right): \Sigma^{\mathrm{C}}\right): \Sigma^{\mathrm{F}}\right]\left(\mathrm{A}_{1}\right): \Sigma^{\mathrm{A}}\right)_{\Phi}\right.\right.$ $\left.\left.\left(\mathrm{A}_{2}:\left[\operatorname{ILL}\left(\mathrm{P}_{1}\right)_{\mathrm{S}}\left(\mathrm{P}_{2}\right)_{\mathrm{A}}\left(\mathrm{C}_{2}:\left[\ldots\left(\mathrm{T}_{2}\right) \ldots\right]\left(\mathrm{C}_{2}\right): \Sigma^{\mathrm{C}}\right): \Sigma^{\mathrm{P}}\right]\left(\mathrm{A}_{2}\right): \Sigma^{\mathrm{A}}\right)_{\Phi}\right]\left(\mathrm{M}_{1}\right)\right)$

Uma primeira dificuldade que se impõe à tentativa de formalizar, segundo a GDF, as classes de aposições não restritivas tal como propostas por Quirk et al. (1985) e Meyer (1992) encontra-se no fato de que estes distinguem as construções apositivas pelo tipo de relação semântica (equivalência, atribuição ou inclusão) que se estabelece entre unidades apositivas referenciais ou não referenciais. A correferencialidade, por exemplo, se estabelece como uma relação de equivalência entre duas unidades apositivas referenciais. A GDF, por outro lado, abriga referência e atribuição no âmbito pragmático (acional), como subatos discursivos, no nível Interpessoal. Note que o termo atribuição em Quirk et al. (1985) e Meyer (1992) concerne a um tipo de relação semântica; na GDF, o termo atribuição (ascription) tem sido usado para traduzir o subato de atribuir ou predicar. Assim, de acordo com a GDF, numa construção apositiva não restritiva com relação semântica de atribuição, por exemplo, a segunda unidade exerceria um subato atributivo em relação à primeira unidade.

${ }^{2}$ Nos índices, Hannay e Keizer (2005) usam números; Hengeveld e Mackenzie (2008) usam letras. Manteremos a formalização utilizada pelos autores.

3 a) $M_{1}=$ um Move; b) $A_{1}=$ um ato discursivo; c) $\Phi=$ a função/papel do ato discursivo dentro do Move (onde se representam as funções discursivas das aposições não restritivas); d) ILL = a Ilocução; os dois atos ilocucionários podem diferir em relação à força ilocucionária (e.g. INT e DECL); e) $\mathrm{P}_{1}=$ participante do ato discursivo; f) $\mathrm{C}_{1}$ = o Conteúdo Comunicado; $\mathrm{T}_{1}=$ um subato atributivo; $\mathrm{R}_{1}=$ um subato referencial; $\Sigma_{1}=$ modificadores lexicais do nível Interpessoal, que podem atuar em diversos níveis (Conteúdo Comunicado - evidentemente, ao que parece, como se vê); Ilocução (francamente, honestamente) e Ato Discursivo (em outras palavras, quer dizer, em todo caso) (HANNAY; KEIZER, 2005, p. 182). 
Se, segundo a GDF, os dois subatos são apenas referir e atribuir, onde tratar as relações de equivalência sinonímica, que não são referenciais, mas também não são atributivas? Em alguns casos, mesmo envolvendo sintagmas nominais, a segunda unidade da construção apositiva se manifesta mais precisamente com o propósito de uma reformulação de natureza metalinguística, em que é oferecida, ao ouvinte/leitor, uma definição para um conceito denotado no sintagma nominal da primeira unidade da construção. ${ }^{4}$ No caso das reformulações, mesmo as que têm caráter metalinguístico, Hannay e Keizer (2005, p. 186) atribuem, à primeira unidade da construção, um valor referencial; mas, à segunda unidade, um valor atributivo. Segue tradução do exemplo fornecido pelos autores: ${ }^{5}$

(1) E Saddam Husseim mesmo em uma declaração confirmou que não haveria negociações e que Iraque está embarcando em uma jihad uma guerra santa. <ICE-GB:S2B-010 \#131:1:A>

Para uma representação da aposição não restritiva em (1) no nível Interpessoal da GDF, Hannay e Keizer (2005, p. 186) propõem:

$\left(\mathrm{M}_{1}:\left[\left(\mathrm{A}_{1}:\left[\operatorname{ILL}\left(\mathrm{P}_{1}\right)_{\mathrm{S}}\left(\mathrm{P}_{2}\right)_{\mathrm{A}}\left(\mathrm{C}_{1}:\left[\ldots\left(\mathrm{T}_{1}\right)\left(\mathrm{R}_{1}\right) \ldots\right]\left(\mathrm{C}_{1}\right): \Sigma^{\mathrm{C}}\right): \Sigma^{\mathrm{F}}\right]\left(\mathrm{A}_{1}\right): \Sigma^{\mathrm{A}}\right)_{\text {Nucl }}\right.\right.$ $\left.\left.\left(\mathrm{A}_{2}:\left[\operatorname{ILL}\left(\mathrm{P}_{1}\right)_{\mathrm{S}}\left(\mathrm{P}_{2}\right)_{\mathrm{A}}\left(\mathrm{C}_{2}:\left[\ldots\left(\mathrm{T}_{2}\right) \ldots\right]\left(\mathrm{C}_{2}\right): \Sigma^{\mathrm{C}}\right): \Sigma^{\mathrm{P}}\right]\left(\mathrm{A}^{2}\right): \Sigma^{\mathrm{A}}\right)_{\text {Simpl }}\right]\left(\mathrm{M}_{1}\right)\right)$

Como a segunda unidade constitui uma opção linguística mais simples e mais acessível de denotar a mesma propriedade que é denotada pela descrição oferecida na primeira unidade da construção apositiva (numa função retórica de simplificação), a denotação relativa às duas unidades é idêntica. Portanto, essas descrições podem ser representadas pela mesma variável x no nível Representacional. ${ }^{6}$ No entanto, as duas unidades diferem tanto nas propriedades atribuídas ao referente (variável f) como nos lexemas usados para expressar essas propriedades. No nível Representacional, Hannay e Keizer (2005, p. 186) propõem a seguinte representação das unidades apositivas nesses casos:

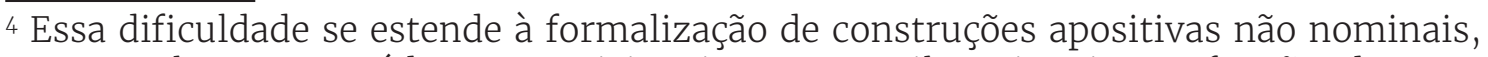
que envolvem conteúdos proposicionais ou atos ilocucionais na função de uma reformulação parafrásica.

5 Tradução de: And Saddam Hussein himself in a statement confirmed that there would be no negotiations and that Iraq is embarking on a jihad a holy war <ICEGB:S2B-010 \#131:1:A> (HANNAY; KEIZER, 2005, p. 186).

${ }^{6}$ A variável associada ao lexema war (guerra) denota, mais apropriadamente, a categoria semântica Episódio (ep).
} 
v. 8 (esp.)

$130-152$ set. 2018

núcleo: $\left(\omega \mathrm{x}_{1}:\left[\left(\left[\omega \mathrm{f}_{1}: \operatorname{Lex}_{\mathrm{N}}\right]\left(\mathrm{f}_{1}\right)\right)\right]\left(\mathrm{x}_{1}\right)\right)$

aposição: $\left(\omega \mathrm{x}_{1}:\left[\left(\left[\omega \mathrm{f}_{2}: \operatorname{Lex}_{\mathrm{N}}\right]\left(\mathrm{f}_{2}\right)\right)\right]\left(\mathrm{x}_{1}\right)\right)$

Keizer (2008, p. 201) defende que as variáveis do nível Representacional não são propriamente entidades, pois a relação entre uma expressão linguística e um elemento extralinguístico é indireta, ou seja, é mediada por um nível de representação mental ou conceptual. Em vez de serem a extensão de um termo, a autora propõe que as variáveis do nível Representacional sejam entendidas como um conjunto de extensão mental do falante de uma descrição (speaker's mental extension set of a description): um conjunto inteiro de entidades mentais às quais, na visão do falante, a(s) propriedade(s) descrita(s) pelo(s) lexema(s) usado(s) em uma expressão se aplica(m).

Para Keizer (2008), considerar a variável do nível Representacional como simbolizando essa extensão mental dos elementos lexicais utilizados numa expressão significa que essa variável não mais representa o referente pretendido, mas uma classe inteira de entidades mentais entre as quais o referente pretendido deve ser selecionado. Esse conjunto de extensão mental não é um conjunto de entidades extralinguísticas. Em vez disso, ele pode ser entendido como uma entidade linguística, posto que faz parte do conhecimento linguístico de longo termo de um participante do discurso, sendo determinado pelas definições dos elementos linguísticos no léxico mental do falante.

A aposição não restritiva em (1) é analisada por Keizer (2008), no nível Representacional, como constituída de duas expressões que, embora apresentem lexemas diferentes, são associadas ao mesmo conjunto de extensão mental, em que as duas descrições se aplicam a um mesmo conjunto de entidades, com a diferença de que a descrição fornecida no segundo elemento da construção é mais familiar, mais acessível ao ouvinte. Com base nessa análise, Keizer (2008, p. 211) propõe a seguinte formalização:

\begin{tabular}{l|l} 
& uma jihad \\
NI: & $\left(\mathrm{A}_{1}: \ldots\left(\mathrm{R}_{1}\right) \ldots\right)_{\text {Nucl }}$ \\
$\mathrm{NR}:$ & $\left(\mathrm{x}_{1}:\left[\mathrm{f}_{1}:\right.\right.$ jihad $)$
\end{tabular}

$$
\mid \begin{aligned}
& \text { uma guerra santa } \\
& \left(\mathrm{A}_{2}:\left(\mathrm{T}_{1}\right)\right)_{\text {Simpl }} \\
& \left(\mathrm{x}_{1}:\left[\mathrm{f}_{2}: \text { guerra }\right]\left[\mathrm{f}_{3}: \text { santa }\right]\right)
\end{aligned}
$$


Para a reformulação contida no exemplo dado ser informativa, o falante instrui o ouvinte a acrescentar jihad à extensão mental que ele já tem de guerra santa. Porém, se o falante julga que o ouvinte não sabe o que significa jihad, nem o que significa guerra santa, as duas informações são vistas como novas, ou seja, como estabelecendo (numa referência construtora) dois conjuntos de extensão mental extensionalmente equivalentes, mas intensionalmente distintos.

Hannay e Keizer (2005, p. 188) aludem, também, à dificuldade de análise da função de rotulação (labelling) por meio de nomes próprios, que não denotam. A solução proposta pelos autores é considerar a existência de um terceiro tipo de subato. Segue o exemplo fornecido por Hannay e Keizer (2005, p. 188):

(2) Tanto a mãe do Sr. Hobson quanto sua ex-esposa, Kay, suplicaram para que ele se rendesse ${ }^{7}$.

Os autores consideram que, nesse tipo de aposição, a construção do Conteúdo Comunicado não se faz por meio de qualquer um dos subatos de referir ou atribuir, mas consiste simplesmente do item lexical, como explicitam a seguir:

$\left(M_{1}:\left[\left(\mathrm{A}_{1}:\left[\operatorname{ILL}\left(\mathrm{P}_{1}\right)_{\mathrm{S}}\left(\mathrm{P}_{2}\right)_{\mathrm{A}}\left(\mathrm{C}_{1}:\left[\ldots\left(\mathrm{T}_{1}\right)\left(\mathrm{R}_{1}\right) \ldots\right]\left(\mathrm{C}_{1}\right): \Sigma^{\mathrm{C}}\right): \Sigma^{\mathrm{F}}\right]\left(\mathrm{A}_{1}\right): \Sigma^{\mathrm{A}}\right)_{\text {Nucl }}\right.\right.$ $\left.\left.\left(\mathrm{A}_{2}:\left[\operatorname{ILL}\left(\mathrm{P}_{1}\right)_{\mathrm{S}}\left(\mathrm{P}_{2}\right)_{\mathrm{A}}\left(\mathrm{C}_{2}: \operatorname{Lex}_{\mathrm{N}}\left(\mathrm{C}_{2}\right)\right): \Sigma^{\mathrm{P}}\right]\left(\mathrm{A}_{2}\right): \Sigma^{\mathrm{A}}\right)_{\mathrm{Lab}}\right]\left(\mathrm{M}_{1}\right)\right)$

A representação traduziria que o Conteúdo Comunicado (C), em vez de se constituir por subatos de referir ou atribuir, restringe-se diretamente pelo lexema representado pelo nome próprio. Além disso, como a aposição toma a forma de nome próprio (de referente dado), e nomes próprios não têm nenhuma denotação, não há nada a ser formalizado no nível Representacional.

Os nomes próprios usados referencialmente são tratados por Hengeveld (2008) como subatos referenciais restringidos pelo nome próprio (lexema) no nível Interpessoal. Keizer (2008), no entanto, argumenta que os nomes próprios podem exercer uma função semântica de restrição. E, embora falhem na atribuição de propriedades, eles correspondem a um conjunto de extensão mental, que abriga todas as

7 Tradução de: Both Mr Hobson's mother and his ex-wife, Kay, had pleaded with him to give himself up. (Hunt ends for most wanted man, The Guardian, 26-07-2004) (HANNAY; KEIZER, 2005, p. 188). 
v. 8 (esp.)

$130-152$ set. 2018

entidades conhecidas por um dado nome. No caso das expressões nominais após a cópula (Eu sou Peter / I am Peter) e nas aposições restritivas, o nome próprio parece, de fato, exercer um tipo especial de atribuição. Voltaremos à questão da representação de nomes próprios nos níveis de formulação da GDF, na seção 3, destinada à formalização das aposições não restritivas em língua portuguesa.

Em coerência com o modelo teórico da GDF, que é orientado da função para a forma, a classificação proposta por Hannay e Keizer (2005) coloca em foco as funções discursivas das aposições não restritivas, mas se restringe a construções que consistem de dois sintagmas nominais. Mesmo admitindo que aposições não nominais possam exercer funções não contempladas na sua proposta, os autores julgam que uma classificação baseada na função é tanto exequível, quanto desejável. Embora concorde que uma classificação baseada nas funções discursivas seja melhor do que alguma baseada na forma sintática (o que é mais recorrente no tratamento da aposição), uma classificação que considere principalmente as funções discursivas talvez revele apenas funções gerais, não privativas das construções apositivas, nem a elas estreitamente peculiares.

Acredito que, tendo em vista a diversidade formal e a multiplicidade funcional das aposições não restritivas, a natureza semântica da relação entre unidades apositivas, um nível intermediário entre forma sintática e função discursiva, parece ser o nível de abstração mais adequado para uma tipologia. Afinal, há menos variantes relativas à natureza da relação semântica estabelecida entre as unidades apositivas do que relativas à forma sintática e às funções discursivas da aposição não restritiva. Por esse motivo, para a classificação das aposições não restritivas, faço a distinção inicial entre dois tipos de relação semântica - equivalência e atribuição.

Seguem, todavia, algumas ressalvas. Primeiro, equivalência e atribuição devem ser vistas em sua natureza textual-semântica, pois elas se instauram na construção do texto, entre segmentos discursivos, como estratégias de textualização, e essa natureza, de fato, aproxima tais relações das funções retóricas tratadas no Nível Interpessoal da GDF. Segundo, retomo aqui a mudança que propus em Nogueira (1999, 2012), de que os subtipos dessas relações, propostos por Quirk et al. (1985) (identificação, reformulação, redenominação, etc) não são propriamente relações semânticas, mas, antes, funções textual-discursivas, já que, claramente, dizem respeito a funções retóricas da expressão apositiva. Por fim, a utilidade descritiva dessa proposta encontra-se na possibilidade de descrever diferentes manifestações da aposição não restritiva, 
relacionando seus aspectos textual-discursivos, textual-semânticos e morfossintáticos, ao tempo em que considera a integração entre esses aspectos. Considero que as diferentes funções textual-discursivas (retóricas) do Nível Interpessoal são instanciadas pelas relações textualsemânticas de equivalência e atribuição, apontadas por Quirk et al. (1985) e Meyer (1992). Ou seja, para cumprimento de algumas funções retóricas, as aposições não restritivas se constituem, no texto, como mecanismos que tipicamente instauram esses dois tipos de relação textual-semântica. Pelas razões acima apontadas, assumo, então, que a diferença quanto ao tipo de relação textual-semântica consista numa primeira e mais ampla distinção das aposições não restritivas. Com base nas diferenças entre aposições referenciais e não referenciais (mas não necessariamente atributivas), em Nogueira (1999, 2012), acomodo os casos de reformulação de natureza metalinguística como uma segunda distinção entre as relações de equivalência: uma equivalência referencial (correferencialidade) e uma equivalência estritamente semântica (sinonímia). A relação de inclusão, apresentada por Quirk et al. (1985) e Meyer (1992), pode ser vista como um tipo de equivalência parcial: a inclusão referencial ocorre na relação entre um Indivíduo, Lugar ou Tempo, que faz parte do conjunto designado na primeira unidade apositiva; e a inclusão semântica ocorre entre unidades lexicais que se relacionam por hiponímia ou meronímia.

Após as mudanças aqui sugeridas, chego ao seguinte quadro de classificação das construções apositivas não restritivas:

Quadro 1 - Tipos de aposições não restritivas

\section{Relação textual-semântica}

Equivalência Referencial Total

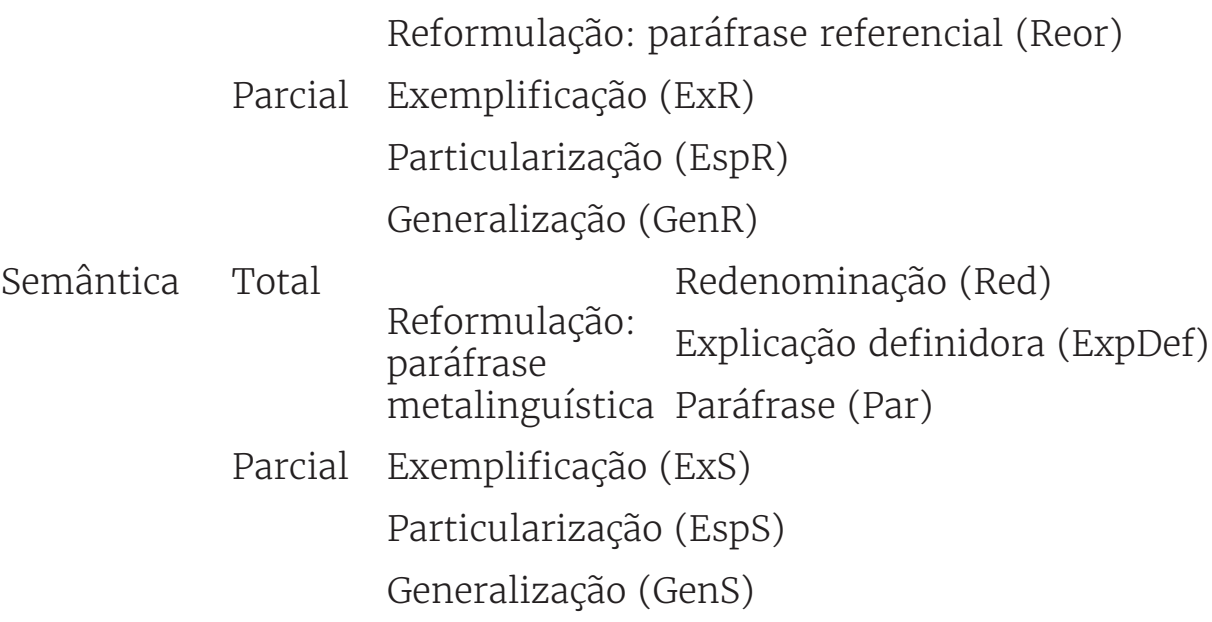

\section{Função textual-discursiva}

Identificação (Ident)

Atribuição

Avaliação - relativa a Indivíduo, Estado-de-coisas, Proposição, Ato Discursivo. (Aval)

Fonte: elaborado pela autora. 
v. 8 (esp.)

130-152 set. 2018

O quadro 1 informa que as aposições não têm apenas natureza referencial e, como estratégia textualizadora de caráter multifuncional, concernem a relações entre segmentos de natureza semântica e estrutura morfossintática bastante diversificadas. Com relação às classificações propostas por Quirk et al. (1985) e Meyer (1992), a nossa sugestão tem a vantagem de tratar a inclusão como uma relação de equivalência parcial, mantendo, portanto, uma disposição mais coerente e simétrica. Com relação à proposta de Hannay e Keizer (2005), nossa proposta tem a vantagem de reunir aposições nominais que não são nem referenciais, nem atributivas; e aposições não nominais, isto é, construções apositivas não restritivas que envolvem segmentos maiores ou menores do que o sintagma nominal, em uma unidade ou em ambas. Na próxima seção, proponho, na formalização de algumas ocorrências que ilustram as categorias da tipologia apresentada no quadro 1, diferenças relativas a seus aspectos textual-semânticos e textual-discursivos.

\section{Análise de aposições não restritivas em língua portuguesa}

Metodologia

Para o exercício de formalização de alguns tipos de aposições não restritivas do quadro 1, utilizo ocorrências identificadas em textos técnico-didáticos, oratórios e dramáticos obtidos do Banco de Dados de Língua Escrita Contemporânea no Brasil, da Faculdade de Ciências e Letras, UNESP de Araraquara-SP. Tais ocorrências já foram analisadas segundo parâmetros sintáticos, textual-semânticos e textualdiscursivos em Nogueira (1999). Recebem, no entanto, neste artigo, a representação de propriedades pragmáticas e semânticas segundo a formalização dos níveis Interpessoal e Representacional da GDF.

Formalização

Segue, nesta seção, a formalização, segundo a GDF, de algumas ocorrências. Essa é uma forma de testar a utilidade descritiva da teoria na explicitação de distinções que julgo relevantes na identificação das classes de aposições não restritivas em língua portuguesa. 
Equivalência

\section{A. Equivalência referencial}

As aposições que se caracterizam pela equivalência referencial entre as unidades apositivas relacionam-se às funções de identificação e reformulação (paráfrase referencial).

a) Identificação (Ident)

A aposição com função de identificação aqui formalizada tem a mesma caracterização que Quirk et al. (1985), Meyer (1992) e Hannay e Keizer (2005) fazem da aposição com função de identificação, particularmente pelo subtipo especificação, em que a descrição contida na primeira unidade indica o papel e a relevância de um referente em um dado discurso, mas não é suficiente para a atribuição da referência pelo ouvinte/leitor.

Destaco que, mais do que o monitoramento da referência, esse tipo de organização da informação na construção apositiva serve ao propósito de orientação argumentativa e de criação de um ambiente de expectativa a partir da primeira unidade da construção.

(3) Um dos mais penetrantes pensadores italianos do nosso tempo, Luigi Pareyson, ao retomar a discussão dos temas centrais da Estética,... (REF-LT).

A representação da aposição em (3) no nível Interpessoal é:

$\left(\mathrm{M}_{\mathrm{I}}:\left[\left(\mathrm{A}_{\mathrm{I}}:\left[\operatorname{ILL}\left(\mathrm{P}_{1}\right)_{\mathrm{S}}\left(\mathrm{P}_{2}\right)_{\mathrm{A}}\left(\mathrm{C}_{\mathrm{I}}:\left[\ldots\left(\mathrm{T}_{\mathrm{I}}\right)\left(-\mathrm{id} \mathrm{R}_{\mathrm{I}}\right) \ldots\right]\left(\mathrm{C}_{\mathrm{I}}\right): \Sigma^{\mathrm{C}}\right): \Sigma^{\mathrm{F}}\right]\left(\mathrm{A}_{\mathrm{I}}\right): \Sigma^{\mathrm{A}}\right)_{\text {Nucl }}\right.\right.$ $\left.\left.\left(\mathrm{A}_{\mathrm{J}}:\left[\operatorname{ILL}\left(\mathrm{P}_{1}\right)_{\mathrm{S}}\left(\mathrm{P}_{2}\right)_{\mathrm{A}}\left(\mathrm{C}_{\mathrm{J}}:\left[\ldots\left(+\mathrm{id} \mathrm{R}_{\mathrm{j}}\right) \ldots\right]\left(\mathrm{C}_{\mathrm{j}}\right): \Sigma^{\mathrm{C}}\right): \Sigma^{\mathrm{P}}\right]\left(\mathrm{A}_{\mathrm{j}}\right): \Sigma^{\mathrm{A}}\right)_{\text {Ident }}\right]\left(\mathrm{M}_{\mathrm{I}}\right)\right)$

Nessa representação, considero que a primeira unidade da construção apositiva é referencial, ainda que indefinida. A segunda unidade, um nome próprio, realiza um subato referencial por meio de uma designação mais específica. No nível Representacional, proponho a seguinte descrição:

$1^{\mathrm{a}}$ unidade: $\left(\mathrm{mx}_{\mathrm{i}}:\left[\left(\mathrm{f}_{\mathrm{i}} \text { : (pensadores }\right)_{\mathrm{N}}\left(\mathrm{f}_{\mathrm{i}}\right)\right)\right.$ : $\left.\left.\left(\mathrm{f}_{\mathrm{j}} \text { : (penetrantes }\right)_{\mathrm{A}}\left(\mathrm{f}_{\mathrm{j}}\right)\left(\mathrm{f}_{\mathrm{i}}\right)\right] \ldots\left(\mathrm{x}_{\mathrm{i}}\right)\right)$ $2^{\mathrm{a}}$ unidade: $\left.\left(\mathrm{x}_{\mathrm{i}} \text { : (Luigi Pareyson) }\right)_{\mathrm{N}}\left(\mathrm{x}_{\mathrm{i}}\right)\right)$ 
v. 8 (esp.)

130-152 set. 2018

Nessa representação, explicito que a primeira unidade da aposição utiliza propriedades (f) para descrição de uma entidade de primeira ordem (x), enquanto a segunda unidade fornece um nome próprio (N), que está associado, como propõe Keizer (2008), a um conjunto de extensão mental, mas não constitui uma propriedade.

b) Paráfrase referencial: reorientação (Reor)

No exemplo (4), há uma ocorrência típica de uma paráfrase referencial (FUCHS, 1982) ou reorientação (MEYER, 1992), em que os dois elementos apositivos são referenciais e identificáveis.

(4) A câmara - a futura máquina fotográfica - já existia e há muito vinha sendo aperfeiçoada. (FOT-LT)

Para o nível Interpessoal, sugiro a seguinte representação:

$\left(M_{\mathrm{I}}:\left[\left(\mathrm{A}_{\mathrm{I}}:\left[\operatorname{ILL}\left(\mathrm{P}_{1}\right)_{\mathrm{S}}\left(\mathrm{P}_{2}\right)_{\mathrm{A}}\left(\mathrm{C}_{\mathrm{I}}:\left[\ldots\left(\mathrm{T}_{\mathrm{I}}\right)\left(+\mathrm{id} \mathrm{R}_{\mathrm{I}}\right) \ldots\right]\left(\mathrm{C}_{\mathrm{I}}\right): \Sigma^{\mathrm{C}}\right): \Sigma^{\mathrm{F}}\right]\left(\mathrm{A}_{\mathrm{I}}\right): \Sigma^{\mathrm{A}}\right)_{\text {Nucl }}\right.\right.$ $\left.\left.\left(A_{\mathrm{J}}:\left[\operatorname{ILL}\left(\mathrm{P}_{1}\right)_{\mathrm{S}}\left(\mathrm{P}_{2}\right)_{\mathrm{A}}\left(\mathrm{C}_{\mathrm{J}}:\left[\ldots\left(+\mathrm{id} \mathrm{R}_{\mathrm{J}}\right) \ldots\right]\left(\mathrm{C}_{\mathrm{J}}\right): \Sigma^{\mathrm{C}}\right): \Sigma^{\mathrm{P}}\right]\left(\mathrm{A}_{\mathrm{J}}\right): \Sigma^{\mathrm{A}}\right)_{\text {reor }}\right]\left(\mathrm{M}_{\mathrm{I}}\right)\right)$

E, para o nível Representacional, o que segue:

$1^{\mathrm{a}}$ unidade: $\left(\mathrm{x}_{\mathrm{i}}:\left(\mathrm{f}_{\mathrm{i}}\right.\right.$ : câmara $\left.\left.\left(\mathrm{f}_{\mathrm{i}}\right)\right)\left(\mathrm{x}_{\mathrm{i}}\right)\right)$

$2^{\mathrm{a}}$ unidade: $\left(\mathrm{x}_{\mathrm{i}}:\left[\left(\mathrm{f}_{\mathrm{j}}:(\text { máquina })_{\mathrm{N}}\left(\mathrm{f}_{\mathrm{J}}\right)\right):\left(\mathrm{f}_{\mathrm{k}}:(\text { fotográfica })_{\mathrm{A}}\left(\mathrm{f}_{\mathrm{k}}\right)\left(\mathrm{f}_{\mathrm{i}}\right)\right):\left(\mathrm{f}_{\mathrm{j}}:(\text { futura })_{\mathrm{A}}\left(\mathrm{f}_{\mathrm{f}}\right)\right)\left(\mathrm{x}_{\mathrm{i}}\right)\right)\right.$

Nessa formalização, explicito que quatro propriedades (f) ajudam a construir a representação da entidade $\mathrm{x}_{\mathrm{i}}$.

A construção apositiva não restritiva a seguir apresenta três unidades, sendo a primeira um pronome de primeira pessoa (eu), segunda, um nome próprio (Mercúrio), e a terceira, uma descrição definida (o mensageiro dos deuses).

(5) Aqui estou eu, Mercúrio, o mensageiro dos deuses, para dizervos. (TEG-LD)

Com relação ao emprego do nome próprio, à semelhança da análise que Keizer (2008, p. 208) propõe para declarações do tipo I am Peter (Eu sou Peter), pergunto-me se a intenção do falante é: a) equacionar duas entidades de primeira ordem numa declaração de identidade ( $\mathrm{x}_{\mathrm{i}}$ 
$=\mathrm{x}_{\mathrm{j}}$ ); ou b) atribuir uma propriedade (f) ao referente do sujeito da oração; c) ou, como sugere Keizer (2008), realizar um tipo especial de atribuição que, em vez de instruir o ouvinte a atribuir uma propriedade a uma entidade, serve para orientá-lo a atribuir o rótulo Mercúrio a uma entidade do discurso já introduzida. O exemplo de aposição não restritiva em (5) me parece uma paráfrase referencial, uma reorientação do referente discursivo. Embora a posição logo após uma referência deiticamente óbvia (pronome de primeira pessoa) sugira que, por não haver necessidade de favorecer a identificação de um referente, o nome próprio não teria a motivação estrita de um subato referencial, mas de um subato atributivo (ligado à extensão mental do rótulo Mercúrio), lembro que o ato de referir não se realiza apenas com os propósitos estritamente referenciais de rastreamento das entidades discursivas.

A reorientação cumpre um papel importante de apresentar, em outra perspectiva, o referente já identificado na primeira unidade. Em outras palavras, o rótulo Mercúrio evoca uma extensão mental supostamente identificável pelo ouvinte, que credencia o enunciador e o compromete com o que vai enunciar. O uso do nome próprio constitui, neste exemplo, a realização de um ato discursivo separado, que, mesmo não estando voltado estritamente para a gestão da referência, tem natureza referencial.

A terceira unidade dessa construção apositiva reapresenta o mesmo referente por meio de uma descrição definida, não apenas evocando propriedades que favoreçam a atribuição da referência, mas construindo, axiologicamente, a identidade dessa entidade discursiva.

A representação, no nível Interpessoal, dessa aposição múltipla seria semelhante à do exemplo (5):

$\left(M_{I}:\left[\left(A_{I}:\left[\operatorname{ILL}\left(P_{1}\right)_{S}\left(P_{2}\right)_{A}\left(C_{I}:\left[\ldots\left(T_{I}\right)\left(+i d R_{I}\right) \ldots\right]\left(C_{I}\right): \Sigma^{C}\right): \Sigma^{F}\right]\left(A_{I}\right): \Sigma^{A}\right)_{\text {Nucl }}\right.\right.$ $\left.\left.\left(\mathrm{A}_{\mathrm{J}}:\left[\operatorname{ILL}\left(\mathrm{P}_{1}\right)_{\mathrm{S}}\left(\mathrm{P}_{2}\right)_{\mathrm{A}}\left(\mathrm{C}_{\mathrm{J}}:\left[\ldots\left(+\mathrm{id} \mathrm{R}_{\mathrm{J}}\right) \ldots\right]\left(\mathrm{C}_{\mathrm{J}}\right): \Sigma^{\mathrm{C}}\right): \Sigma^{\mathrm{P}}\right]\left(\mathrm{A}_{\mathrm{J}}\right): \Sigma^{\mathrm{A}}\right)_{\text {reor }}\right]\left(\mathrm{M}_{\mathrm{I}}\right)\right)$ $\left.\left.\left(\mathrm{A}_{\mathrm{k}}:\left[\operatorname{ILL}\left(\mathrm{P}_{1}\right)_{\mathrm{S}}\left(\mathrm{P}_{2}\right)_{\mathrm{A}}\left(\mathrm{C}_{\mathrm{k}}:\left[\ldots\left(+\mathrm{id} \mathrm{R}_{\mathrm{J}}\right) \ldots\right]\left(\mathrm{C}_{\mathrm{k}}\right): \Sigma^{\mathrm{C}}\right): \Sigma^{\mathrm{P}}\right]\left(\mathrm{A}_{\mathrm{k}}\right): \Sigma^{\mathrm{A}}\right)_{\text {reor }}\right]\left(\mathrm{M}_{\mathrm{I}}\right)\right)$

No nível Representacional, proponho a seguinte formalização:

$1^{\mathrm{a}}$ unidade: $\left(\mathrm{x}_{\mathrm{i}}: \varnothing\left(\mathrm{x}_{\mathrm{i}}\right)\right)$

$2^{\mathrm{a}}$ unidade: $\left(\mathrm{x}_{\mathrm{i}}\right.$ : Mercúrio $\left._{\mathrm{N}}\left(\mathrm{x}_{\mathrm{i}}\right)\right)$

$3^{\mathrm{a}}$ unidade: $\left.\left(\mathrm{x}_{\mathrm{i}}:\left[\left(\mathrm{f}_{\mathrm{i}}:\left[\left(\mathrm{f}_{\mathrm{j}} \text { :mensageiro }\right)_{\mathrm{N}}\left(\mathrm{f}_{\mathrm{j}}\right)\right)\left(\mathrm{mx}_{\mathrm{j}}:\left[\left(\mathrm{f}_{\mathrm{k}}:(\text { deuses })_{\mathrm{N}}\left(\mathrm{f}_{\mathrm{k}}\right)\right)\left(\mathrm{x}_{\mathrm{j}}\right)_{\varphi}\right]\right)_{\mathrm{Ref}}\right]\left(\mathrm{f}_{\mathrm{i}}\right)\right)\left(\mathrm{x}_{\mathrm{i}}\right)_{\varphi}\right]\right)$ 
v. 8 (esp.)

130-152 set. 2018
A formalização explicita, com base em Hengeveld (2008, p. 53), que o pronome, usado na unidade núcleo, denota uma entidade de primeira ordem $\left(\mathrm{x}_{\mathrm{i}}\right)$, mas não como fazem os itens lexicais, já que esse pronome não tem nenhum sentido em si mesmo. A segunda unidade denota a mesma entidade $\left(\mathrm{x}_{\mathrm{i}}\right)$, por meio de um nome próprio. Já na terceira unidade apositiva, a mesma entidade é denotada por meio de uma propriedade (mensageiro) modificada por uma propriedade configuracional (dos deuses).

B. Equivalência semântica

B.1 Reformulação metalinguística

a) Redenominação

O exemplo a seguir ilustra a função de reformulação metalinguística em que a segunda unidade apresenta-se como um lexema alternativo para a denotação de um conceito já apresentado na primeira unidade.

(6) Antes de nomear um embaixador, é necessário obter a aprovação da pessoa indicada pelo governo, que vai recebêlo. Faz-se o pedido de agreement, ou anuência. (DIP-LT)

À semelhança do que Hannay e Keizer (2005) propõem para a função de rotulação (labelling), nos casos de reformulação metalinguística, a representação deve traduzir que o Conteúdo Comunicado, em vez de se constituir por atos de referir e atribuir, restringe-se diretamente pelo lexema apresentado como designação mais fácil e mais familiar ao ouvinte/leitor.

$\left(M_{\mathrm{I}}:\left[\left(\mathrm{A}_{\mathrm{I}}:\left[\operatorname{ILL}\left(\mathrm{P}_{1}\right)_{\mathrm{S}}\left(\mathrm{P}_{2}\right)_{\mathrm{A}}\left(\mathrm{C}_{\mathrm{I}}:\left[\ldots\left(\mathrm{T}_{\mathrm{I}}\right)\left(\mathrm{R}_{\mathrm{I}}\right) \ldots\right]\left(\mathrm{C}_{\mathrm{I}}\right): \Sigma^{\mathrm{C}}\right): \Sigma^{\mathrm{F}}\right]\left(\mathrm{A}_{\mathrm{I}}\right): \Sigma^{\mathrm{A}}\right)_{\text {Nucl }}\right.\right.$ $\left.\left.\left(\mathrm{A}_{\mathrm{J}}:\left[\operatorname{ILL}\left(\mathrm{P}_{1}\right)_{\mathrm{S}}\left(\mathrm{P}_{2}\right)_{\mathrm{A}}\left(\mathrm{C}_{\mathrm{J}}: \operatorname{Lex}_{\mathrm{N}}\left(\mathrm{C}_{\mathrm{J}}\right)\right): \Sigma^{\mathrm{P}}\right]\left(\mathrm{A}_{\mathrm{J}}\right): \Sigma^{\mathrm{A}}\right)_{\text {Red }}\right]\left(\mathrm{M}_{\mathrm{I}}\right)\right)$

Outra possibilidade de representação é considerar que, nesses casos de reformulação, como sugere Keizer (2008, p. 210), a primeira unidade da aposição realize um subato referencial, e a segunda, um subato de atribuição, sendo as duas unidades interpretadas como relativas ao mesmo conjunto de extensão mental, isto é, como descrições alternativas aplicáveis ao mesmo conjunto de entidades. Segue a representação: 


\begin{tabular}{|c|c|c|}
\hline & agreement & anuência \\
\hline NI: & $\left(\mathrm{A}_{\mathrm{I}}: \ldots\left(\mathrm{R}_{\mathrm{I}}\right) \ldots\right)_{\text {Nucl }}$ & $\left(A_{J}:\left(T_{J}\right)\right)_{S i m p}$ \\
\hline
\end{tabular}

Seguindo Hannay e Keizer (2005), analiso que a segunda unidade da construção apositiva consiste numa alternativa de denotar, por meio de uma propriedade $\left(f_{j}\right)$, o mesmo estado-de-coisas que é denotado pela propriedade $\left(f_{i}\right)$, usada na primeira unidade.

$1^{\text {a }}$ unidade: $\left(e_{\mathrm{i}}:\left[\left(\left[\mathrm{f}_{\mathrm{i}}\right.\right.\right.\right.$ : agreement $\left.\left.\left.]\left(\mathrm{f}_{\mathrm{i}}\right)\right)\right]\left(\mathrm{e}_{\mathrm{i}}\right)\right)$ $2^{\mathrm{a}}$ unidade: $\left(e_{\mathrm{i}}:\left[\left(\left[\mathrm{f}_{\mathrm{j}}\right.\right.\right.\right.$ : anuência $\left.\left.\left.]\left(\mathrm{f}_{\mathrm{j}}\right)\right)\right]\left(\mathrm{e}_{\mathrm{i}}\right)\right)$

Nessa interpretação, podemos dizer que a relação textualsemântica de equivalência está presente entre as unidades da aposição, ainda que a segunda unidade da construção desempenhe, do ponto de vista pragmático, um tipo particular de subato de atribuição (predicação).

Por esse motivo, embora as funções retóricas sejam diferentes, a denotação das duas descrições é aqui considerada idêntica, tal como no caso da reformulação. Novamente, a mesma variável (e) é aplicada no nível Representacional, também com a diferença apenas relativa à variável propriedade (f).

b) Explicação definidora

O exemplo em (7) ilustra o uso de aposições não restritivas com função de reformulação metalinguística em explicações definidoras.

(7) Nem mesmo a puberdade - início da adolescência - é período crítico, mas natural. (AE-LT)

De acordo com a proposta de Hannay e Keizer (2005), numa construção apositiva com função de reformulação, a primeira unidade é tipicamente interpretada como um subato de referência, enquanto o segundo elemento é analisado como um subato de atribuição. Segue a representação dessa análise:

$\left(M_{\mathrm{I}}:\left[\left(\mathrm{A}_{\mathrm{I}}:\left[\operatorname{ILL}\left(\mathrm{P}_{1}\right)_{\mathrm{S}}\left(\mathrm{P}_{2}\right)_{\mathrm{A}}\left(\mathrm{C}_{\mathrm{I}}:\left[\ldots\left(\mathrm{T}_{\mathrm{I}}\right)\left(\mathrm{R}_{\mathrm{I}}\right) \ldots\right]\left(\mathrm{C}_{\mathrm{I}}\right): \Sigma^{\mathrm{C}}\right): \Sigma^{\mathrm{F}}\right]\left(\mathrm{A}_{\mathrm{I}}\right): \Sigma^{\mathrm{A}}\right)_{\text {Nucl }}\right.\right.$ $\left.\left.\left(\mathrm{A}_{\mathrm{J}}:\left[\operatorname{ILL}\left(\mathrm{P}_{1}\right)_{\mathrm{S}}\left(\mathrm{P}_{2}\right)_{\mathrm{A}}\left(\mathrm{C}_{\mathrm{J}}:\left[\ldots\left(\mathrm{T}_{\mathrm{J}}\right) \ldots\right]\left(\mathrm{C}_{\mathrm{J}}\right): \Sigma^{\mathrm{C}}\right): \Sigma^{\mathrm{P}}\right]\left(\mathrm{A}_{\mathrm{J}}\right): \Sigma^{\mathrm{A}}\right)_{\text {Expl Def }}\right]\left(\mathrm{M}_{\mathrm{I}}\right)\right)$ 
v. 8 (esp.)

130-152 set. 2018

No nível Representacional, as duas unidades são analisadas como tendo o mesmo conjunto de extensão mental, e as duas descrições aplicam-se a um mesmo conjunto de entidades (e), com a diferença de que a descrição fornecida no segundo elemento da construção é mais acessível ao ouvinte.

$1^{\mathrm{a}}$ unidade: $\left(\mathrm{e}_{\mathrm{i}}:\left[\left(\left[\mathrm{f}_{\mathrm{i}}\right.\right.\right.\right.$ : puberdade $\left.\left.\left.]\left(\mathrm{f}_{\mathrm{i}}\right)\right)\right]\left(\mathrm{e}_{\mathrm{i}}\right)\right)$

$2^{\mathrm{a}}$ unidade: $\left(\mathrm{e}_{\mathrm{i}}:\left[\left(\left[\mathrm{f}_{\mathrm{j}}\right.\right.\right.\right.$ : início da adolescência $\left.\left.\left.]\left(\mathrm{f}_{\mathrm{j}}\right)\right)\right]\left(\mathrm{e}_{\mathrm{i}}\right)\right)$

Novamente, a relação de equivalência se mantém no nível textual-semântico, mesmo sendo a segunda unidade da aposição um subato atributivo.

c) Paráfrase

Na aposição em (8), há uma paráfrase entre segmentos não nominais.

(8) Caberia à faculdade do gosto perceber quando a síntese foi alcançada, istoé, quando o artista produziu uma bela representação da existência, ou quando malogrou no seu intento. (REF-LT)

Uma representação, no nível Interpessoal, para esse tipo de aposição deve revelar que a paráfrase se manifesta entre dois Conteúdos Comunicados:

$\left(M_{\mathrm{I}}:\left[\left(\mathrm{A}_{\mathrm{I}}:\left[\operatorname{ILL}\left(\mathrm{P}_{1}\right)_{\mathrm{S}}\left(\mathrm{P}_{2}\right)_{\mathrm{A}}\left(\mathrm{C}_{\mathrm{I}}:\left[\ldots\left(\mathrm{T}_{\mathrm{I}}\right)\left(\mathrm{R}_{\mathrm{I}}\right) \ldots\right]\left(\mathrm{C}_{\mathrm{I}}\right): \Sigma^{\mathrm{C}}\right): \Sigma^{\mathrm{F}}\right]\left(\mathrm{A}_{\mathrm{I}}\right): \Sigma^{\mathrm{A}}\right)_{\text {Nucl }}\right.\right.$ $\left(\mathrm{A}_{\mathrm{J}}:\left[\operatorname{ILL}\left(\mathrm{P}_{1}\right)_{\mathrm{S}}\left(\mathrm{P}_{2}\right)_{\mathrm{A}}\left(\mathrm{C}_{\mathrm{J}}:\left[\ldots\left(\mathrm{T}_{\mathrm{J}}\right)\left(\mathrm{R}_{\mathrm{J}}\right) \ldots\right]\left(\mathrm{C}_{\mathrm{J}}\right): \Sigma^{\mathrm{C}} \operatorname{par}\right): \Sigma^{\mathrm{F}}\right]\left(\mathrm{A}_{\mathrm{J}}\right): \Sigma^{\mathrm{A}}\right)$

Em (8), afirma-se que a faculdade do gosto torna o indivíduo capaz de perceber (concluir) quando a síntese foi alcançada, que equivale a julgar quando o artista produziu uma bela representação da existência. A formalização deve informar a existência de uma relação de equivalência, estabelecida no contexto, entre dois Conteúdos Proposicionais (p), pois analiso esses segmentos como constructos mentais. Segue a representação da aposição em (8) no nível Representacional:

$1^{\mathrm{a}}$ unidade: $\left(\mathrm{p}_{\mathrm{i}}\right.$ : [a síntese foi alcançada] $\left.\left(\mathrm{p}_{\mathrm{i}}\right)\right)$

$2^{\mathrm{a}}$ unidade: ( $\mathrm{p}_{\mathrm{i}}$ : [o artista produziu uma bela representação da existência] $\left(\mathrm{p}_{\mathrm{i}}\right)$ ) 
Atribuição

a) Avaliação relativa a Indivíduo

No exemplo (9), a relação textual-semântica entre as unidades é de atribuição e, nesse caso, a segunda unidade apositiva expressa uma avaliação do autor a respeito da entidade designada na primeira unidade apositiva. Esta função se aproxima da função discursiva de justificação, proposta por Hannay e Keizer (2005).

(9) Maureen Bisilliat - uma excelente fotógrafa brasileira - filmou e fotografou as tribos indígenas no Xingu. (FOT-LT).

Na representação do exemplo acima, explicito a informação de que a segunda unidade apositiva exerce um subato atributivo:

$\left(\mathrm{M}_{\mathrm{I}}:\left[\left(\mathrm{A}_{\mathrm{I}}:\left[\operatorname{ILL}\left(\mathrm{P}_{1}\right)_{\mathrm{S}}\left(\mathrm{P}_{2}\right)_{\mathrm{A}}\left(\mathrm{C}_{\mathrm{I}}:\left[\ldots\left(\mathrm{T}_{\mathrm{I}}\right)\left(+\mathrm{id} \mathrm{R}_{\mathrm{I}}\right) \ldots\right]\left(\mathrm{C}_{\mathrm{I}}\right): \Sigma^{\mathrm{C}}\right): \Sigma^{\mathrm{F}}\right]\left(\mathrm{A}_{\mathrm{I}}\right): \Sigma^{\mathrm{A}}\right)_{\text {Nucl }}\right.\right.$ $\left.\left.\left(\mathrm{A}_{\mathrm{J}}:\left[\operatorname{ILL}\left(\mathrm{P}_{1}\right)_{\mathrm{S}}\left(\mathrm{P}_{2}\right)_{\mathrm{A}}\left(\mathrm{C}_{\mathrm{J}}:\left[\ldots\left(\mathrm{T}_{\mathrm{J}}\right) \ldots\right]\left(\mathrm{C}_{\mathrm{J}}\right): \Sigma^{\mathrm{C}}\right): \Sigma^{\mathrm{P}}\right]\left(\mathrm{A}_{\mathrm{J}}\right): \Sigma^{\mathrm{A}}\right)_{\mathrm{Aval}}\right]\left(\mathrm{M}_{\mathrm{I}}\right)\right)$

No nível Representacional, explicito que as unidades apositivas se aplicam a uma mesma entidade $\mathrm{x}_{\mathrm{i}}$ (Indivíduo).

$1^{\mathrm{a}}$ unidade: $\left(\mathrm{x}_{\mathrm{i}}\right.$ : Maureen Bisilliat $\left._{\mathrm{N}}\left(\mathrm{x}_{\mathrm{i}}\right)\right)$

$2^{a}$ unidade: $\left(\mathrm{x}_{\mathrm{i}}\right.$ : $\left[\left(\mathrm{f}_{\mathrm{i}}\right.\right.$ : fotógrafa $\left.\mathrm{N}_{\mathrm{N}}\left(\mathrm{f}_{\mathrm{i}}\right)\right):\left(\mathrm{f}_{\mathrm{j}}\right.$ : excelente $_{\mathrm{A}}\left(\mathrm{f}_{\mathrm{j}}\right):\left(\mathrm{f}_{\mathrm{k}}\right.$ : brasileira $\left.\left.\left._{\mathrm{A}}\left(\mathrm{f}_{\mathrm{k}}\right)\right)\right]\left(\mathrm{x}_{\mathrm{i}}\right)\right)$

b) Avaliação em relação a um Conteúdo Proposicional

Em (10), uma aposição não restritiva de valor atributivo tem função de avaliação que incide sobre um Conteúdo Proposicional. Tratase de um exemplo típico do que as gramáticas tradicionais de língua portuguesa designam como aposto de oração.

(10) Portanto, aimportânciaeo tamanhodo país em nada influenciam a precedência dada a seus representantes diplomáticos: uma noção que não podemos esquecer. (DIP-LT)

Segue a representação, no nível Interpessoal, da aposição não restritiva em (10): 
v. 8 (esp.)

$130-152$ set. 2018

$\left(\mathrm{M}_{\mathrm{I}}:\left[\left(\mathrm{A}_{\mathrm{I}}:\left[\operatorname{ILL}\left(\mathrm{P}_{1}\right)_{\mathrm{S}}\left(\mathrm{P}_{2}\right)_{\mathrm{A}}\left(\mathrm{C}_{\mathrm{I}}:\left[\ldots\left(\mathrm{T}_{\mathrm{I}}\right)\left(\mathrm{R}_{\mathrm{I}}\right) \ldots\right]\left(\mathrm{C}_{\mathrm{I}}\right): \Sigma^{\mathrm{C}}\right): \Sigma^{\mathrm{F}}\right]\left(\mathrm{A}_{\mathrm{I}}\right): \Sigma^{\mathrm{A}}\right)_{\text {Nucl }}\right.\right.$ $\left.\left(A_{J}:\left[\operatorname{ILL}\left(P_{1}\right)_{S}\left(P_{2}\right)_{A}\left(C_{J}:\left[\ldots\left(T_{J}\right) \ldots\right]\left(C_{J}\right): \Sigma^{C}\right): \Sigma^{F}\right]\left(A_{J}\right): \Sigma^{A}\right)_{\text {Aval }}\right)$

E, para a representação no nível Representacional, sugiro:

$1^{\text {a }}$ unidade: $\left(\mathrm{p}_{\mathrm{i}}:\left([\ldots\right.\right.$ lex...] $\left.)\left(\mathrm{p}_{\mathrm{i}}\right)\right)$

$2^{\mathrm{a}}$ unidade: $\left(\mathrm{p}_{\mathrm{i}}:\left(\mathrm{f}_{\mathrm{i}}:\right.\right.$ noção $\mathrm{o}_{\mathrm{N}}\left(\mathrm{f}_{\mathrm{i}}\right): \mathrm{f}_{\mathrm{j}}:\left(\mathrm{x}_{\mathrm{j}}:(\ldots \text { lex } \ldots)_{\mathrm{A}}\left(\mathrm{x}_{\mathrm{j}}\right)\left(\mathrm{f}_{\mathrm{j}}\right)\right)$

Nessa formalização, informo que um Conteúdo Proposicional (p) é encapsulado pelo nome noção, que designa uma Propriedade $\left(\mathrm{f}_{\mathrm{i}}\right)$.

\section{Considerações finais}

Neste artigo, retomei o tema das construções apositivas não restritivas para, à luz da Gramática Discursivo-Funcional (GDF), propor uma classificação dessas aposições a partir de uma correlação entre natureza da relação textual-semântica entre as unidades apositivas e suas funções textual-discursivas. Nessa classificação, abrigo aposições não restritivas nominais e não nominais, embora o critério essencial tenha sido o da distinção textual-semântica entre relações de equivalência e atribuição.

A principal motivação para a escolha do suporte teórico da GDF encontra-se na atenção e na abordagem que Hannay e Keizer (2005) e Keizer (2008) deram às aposições, em particular às aposições não restritivas, destacando-as como Atos Discursivos autônomos, que contribuem com seu próprio valor de verdade e força ilocucional para a construção discursiva. O exercício de analisar e formalizar ocorrências concretas dessas construções segundo as categorias dos níveis Interpessoal e Representacional da GDF impõe, sem dúvida, atenção e rigor na tentativa de explicitar, por meio de uma formalização diferente, fatos linguísticos que considero empiricamente diferentes e relevantes para uma proposta tipológica.

Com esse exercício teórico, espero ter demonstrado as relações que vejo entre as distinções textual-semânticas e textual-discursivas que apresento na tipologia de aposições não restritivas do quadro 1. De acordo com ela, a relação de equivalência, semântica ou referencial, deve ser entendida como opção que tipicamente instancia as funções textual-discursivas de identificação, de reformulação em paráfrases metalinguísticas ou referenciais, de redenominação (numa equivalência total) e as funções de exemplificação, particularização e generalização 
(numa equivalência parcial); já a relação de atribuição entre as unidades apositivas é opção que tipicamente instancia a função textual-discursiva de avaliação em relação a diferentes tipos de entidades, tais como Indivíduos, Estados-de-coisas, Conteúdos Proposicionais.

Cumpre dizer, ainda, que a correspondência aqui detalhada entre relações textual-semânticas e funções textual-discursivas me parece natural e motivada, mas não é automática. Além disso, sendo estratégia textualizadora de natureza multifuncional, as aposições não restritivas podem manifestar uma sobreposição de funções textual-discursivas. É possível, por exemplo, que a função textual-discursiva de avaliação se manifeste numa relação textual-semântica de equivalência referencial como expediente de reorientação argumentativo-atitudinal de um referente discursivo já identificado na primeira unidade da construção.

\section{Referências}

BLAKEMORE, Diane. Are apposition markers discourse markers? In: Journal of Linguistics, 32, Cambridge: Cambridge University Press, 1996.

CAMARA JR., Joaquim M. Dicionário de linguística e gramática. 13. ed. Petrópolis: Vozes, 1986.

DIK, Simon C. The Theory of Functional Grammar, vol. 1, ed. by HENGEVELD (Kees). Berlin/New York: Mouton de Gruyter, 1997.

FUCHS, C. La paraphrase. Paris: Presses universitaires de France, 1982.

HANNAY, Mike; KEIZER, Evelien. A discourse treatment of english nonrestrictive nominal appositions in Functional Discourse Grammar. In: GÓMEZ-GONZÁLEZ, María de los Ángeles; MACKENZIE, J. Lachlan. Studies in Functional Discourse Grammar, 26, 2005. p. 159-194.

HENGEVELD, Kees; MACKENZIE, J. Lachlan. Functional Discourse Grammar: a typologically-based theory of language structure. Oxford: Oxford University Press, 2008.

HENGEVELD, Kees. Prototypical and non-prototypical noun phrases in Functional Discourse Grammar. In: VELASCO, Daniel G.; RIJKHOFF, Jan. The noun phrase in Functional Discourse Grammar. Berlin, Mouton de Gruyter, 2008. p. 43-62.

KEIZER, Evelien. Reference and ascription in Functional Discourse Grammar: An inventory of problems and some possible solutions. In: VELASCO, Daniel G.; RIJKHOFF, Jan. The noun phrase in Functional Discourse Grammar. Berlin, Mouton de Gruyter, 2008. p. 181-220.

MATTHEWS, P.H. Juxtaposition. In: Syntax. New York: Cambridge University Press, 1981. p. 220-241. 
v. 8 (esp.)

$130-152$ set. 2018

MEYER, Charles F. Apposition in contemporary English. New York: Cambridge University Press, 1992.

NOGUEIRA, Márcia T. A aposição não restritiva em textos do português contemporâneo escrito no Brasil (Tese de doutorado). Araraquara: UNESP, 1999.

. Propriedades textual-discursivas da aposiçãa não restritiva. In: SOUZA, Edson R. Funcionalismo linguístico: análise e descrição. São Paulo: Contexto, 2012. p. 147-169.

QUIRK, R. et al. A comprehensive grammar of the English language. London/New York: Longman, 1985. 